\section{Visión Electrónica Más que un estado sólido \\ https://doi.org/10.14483/issn.2248-4728}

UNIVERSIDAD DISTRITAL

FRANCISCO JOSÉ DE CALDAS

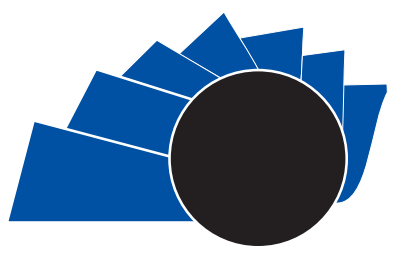

VISIÓN ELECTRONICA

Visión de caso

\title{
Automatización de un taladro de árbol para madera
}

\author{
Automation of a drill wood tree
}

Joseph Anthony Carrera Carrera ${ }^{1}$, Baldomero Méndez Pallares ${ }^{2}$

\section{INFORMACIÓN DEL ARTICULO}

Historia del articulo Enviado: 19/12/2017

Recibido: 16/03/2018

Aceptado: 26/07/2018

\section{RESUMEN}

El presente documento describe una alternativa de diseño para automatizar un taladro de árbol, teniendo en cuenta las características funcionales: posicionamiento, sistema de alimentación, profundidad de taladrado, y el marcado de cada una de las piezas trabajadas. Con la automatización del taladro y sus diferentes estaciones se pretende aumentar la productividad, garantizando una mejor calidad y brindándole al operario un menor esfuerzo físico, ya que éste solo requerirá suministrar la materia prima al sistema de alimentación, además de monitorear y supervisar el proceso. Se muestran los bocetos y diseños obtenidos, además de los componentes y mecanismos seleccionados para lograr integrar los procesos de manufactura que implica el automatizar este proceso

\section{Keywords:}

Automation

Wooding industry

Manufacture

Mechanism

Drilling wood tree

\section{ABSTRACT:}

The present document shows alternative of design to automate a drill wood tree, given the function characteristics: positioning, the feeding system, the deepness of drilling and the marking of each of these worked pieces. With the automation of the drill and it different stations, this intention is to increase the productive, warranting a better quality and offering to the operator less physic effort, already it only required supply the raw material to the feeding system, also to monitoring and supervise this process. It shown us the sketches and designs proposed, also the components and mechanism selected to achieve integrate the manufacturing process that implying to automation this process.

\footnotetext{
1 Ingeniero Mecatrónico, Universidad Piloto de Colombia, Colombia. Miembro del semillero de Investigación en Automatización y Domótica. Correo electrónico: josephcarrera@upc.edu.co. ORCID: https://orcid.org/0000-0002-3743-1467.

2Ingeniero Mecánico, Universidad INCCA de Colombia, Colombia. Especialista en Mecatrónica, Tecnológica Industrial Colombiana, Colombia. MSc. En Ingeniería de Procesos Industriales, Universidad de Ibagué, Colombia. Docente Universidad Piloto de Colombia, Colombia. Miembro del grupo de investigación INNOVATIC Colombia. Correo electrónico: baldomero-mendez@upc.edu.co. ORCID: $\underline{\text { https://orcid.org/0000-0002-7960-0433. }}$ 


\section{Introducción}

En la actualidad los diseños de productos o dispositivos que se comercializan, requieren una serie de procesos de manufactura para cada uno de sus componentes, algunos de ellos conllevan al mecanizado de agujeros con el fin de sujetarlos a otros componentes. Ese mecanizado por lo general lo realiza un taladro de tipo árbol a nivel industrial.

Este tipo de taladro se opera de manera manual, lo que influye algunas veces en un retraso en la producción debido a varios factores, como por ejemplo: fatiga del operario, ubicación y sujeción de la pieza a la hora de realizar el agujero, precisión, entre otras.

En el caso que nos ocupa, en la industria maderera este proceso es uno de los más utilizados para sujetar piezas de madera a través de tornillos. En la manufactura de parques infantiles se usa la madera denominada "Pino Pátula” - altamente resistente y donde se requiere del mecanizado de agujeros, pues la estructura de un parque se hace de este material en diferentes diámetros y longitudes, o uniendo piezas entre sí.

Un recorrido por algunos trabajos realizados en torno a la automatización de este tipo de procesos, arroja una idea sobre la vigencia $y$, por tanto, la necesidad de abordar la investigación sobre el proceso de Taladrado. Inicialmente, en la Universidad Simón Bolívar -Venezuela-, se desarrolló el diseño de una máquina automatizada perforadora de tablas cuyo diseño era capaz de realizar el taladrado de tablas de madera, tanto horizontal como verticalmente, adaptable a una banda transportadora y de poca intervención humana para su operación, figura 1. Además, se evidencia un bajo costo de fabricación y altas posibilidades de elaboración en el país, [1]. Su principio de funcionamiento está definido por la siguiente secuencia: La tabla toca la tranca que está en la salida de la máquina activando un sensor de posición mecánica. Éste le da la señal a los pistones de la tranca de la entrada haciendo que descienda e impida que entre otra tabla. Al mismo tiempo se activa el motor neumático que mueve las barras roscadas haciendo que las guías de centrado fijen la tabla en la posición central de la máquina. En ese momento se activan los cilindros que mueven las guías de los taladros horizontales, realizando la perforación de los costados de la pieza. Cuando se retiran estos taladros volviendo a su posición original comienza a descender la estructura superior de los taladros verticales, y se realiza el perforado vertical y luego comienzan a ascender. En ese momento ocurre el enganche con la tranca de la salida liberando la tabla y desactivándose el sensor de posición mecánica. Con esto se les da la señal a los cilindros de la barra en la entrada de subir y al motor neumático de girar en sentido contrario para soltar la pieza que estaba sujetando en el centro [1].

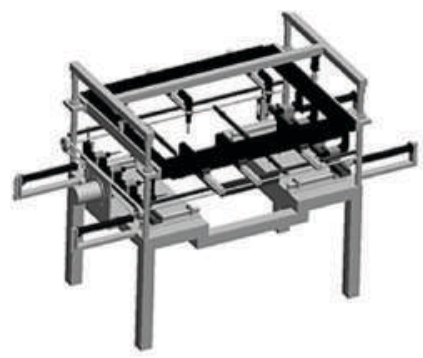

Figura 1. Máquina Taladradora [1].

Otro estudio, realizado en el Instituto Politécnico Nacional -Escuela Superior de Ingeniería Mecánica y Eléctrica- en México, desarrolla la Automatización de un taladro para perforar papel, teniéndose por objeto mejorar la productividad de una empresa, mejorar las condiciones de trabajo del personal, realizar operaciones difíciles, simplificar el mantenimiento, integrar la gestión y producción, y mejorar la calidad del producto, [2]. El autor propone automatizar este proceso de perforado, con tecnología neumática, el operador solo tendrá que poner los calendarios en el alimentador y recogerlos del otro lado en el recibidor. Un actuador neumático 1 colocara el calendario en posición, un sensor óptico de color $\mathrm{S} 1$ verificara que este puesto de forma correcta, otro actuador 2 sujetara para que no se mueva al ser perforado, un actuador 3 perfora el producto y un último actuador 4 lo expulsa de la bancada cayendo al recibidor. Los actuadores utilizan sensores de posición para los diferentes procesos; el sistema de control es basado en controladores lógicos programables (PLC), utilizando lenguaje Ladder, que de acuerdo a la norma IEC 61 131-3, es un lenguaje grafico que se asemeja en muchos aspectos al desarrollo de secuencias en lógica cableada, cada una de las partes del programa se representa en una rama compuesta de contactos y bobinas En la figura 2 se muestra un bosquejo del proceso.

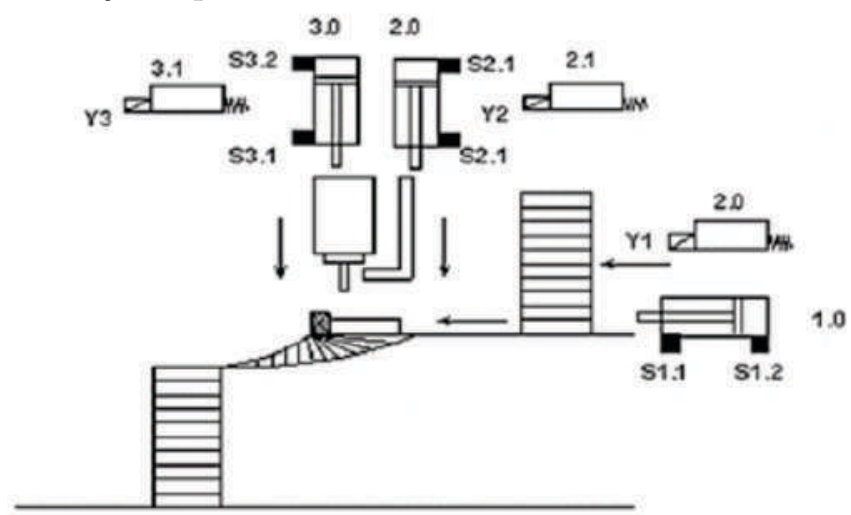

Figura 2. Esquema del proceso [2]. 
De otro lado, en la Universidad Tecnológica de Pereira -Colombia-, se desarrolló el diseño de un Sistema de Control para un taladro Electroneumático de banco, bajo la norma GEMMA y Ambiente SCADA, figura 3, [3]. Una descripción básica del funcionamiento del sistema electroneumático de taladro de la siguiente forma: un estado inicial: Taladro neumático desactivado, M Desactivado. Cilindro de simple efecto desactivado, $\mathrm{C}$ Desactivado. Cilindro de doble efecto posición inicial (hacia la derecha), ao Activado. Actuador lineal posición inicial (hacia arriba), bo Parte funcional: Inicialmente se ubica un objeto no metálico por un conducto, el cual será detectado por $\mathrm{d} 1$, luego se activará el cilindro de doble efecto (a+ pasando de suposición inicial a la posición final accionando a 1) desplazando el objeto de un lugar 1 (debajo del conducto) a un lugar 2 (debajo del taladro electro-neumático); después, el objeto accionará d2, luego se activará el actuador lineal $(b+$ pasando de su posición inicial a la posición final) al mismo tiempo el taladro neumático (M)se activa; cuando el actuador lineal llega a su posición final activa b1, desactiva M, y el actuador lineal vuelve a su posición inicial; finalmente se activa el cilindro de simple efecto $\mathrm{C}$ y el cilindro de doble efecto vuelve a su posición inicial, [3].

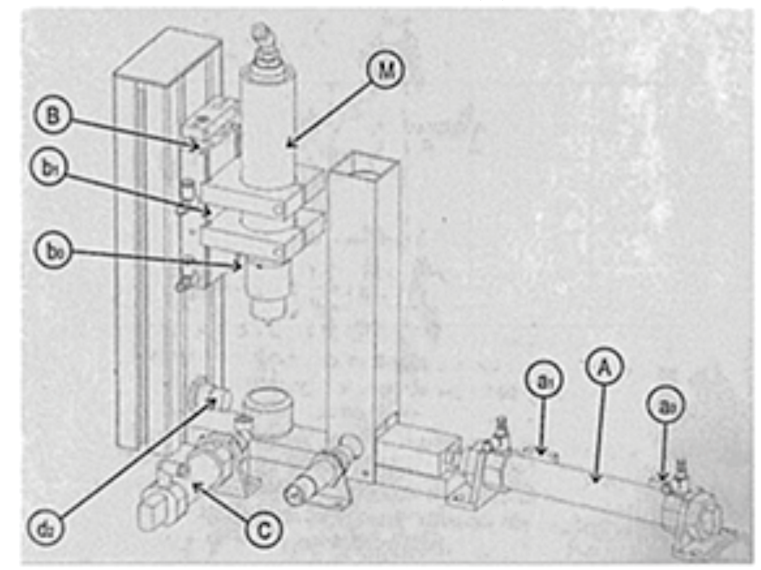

Figura 3. Esquema del proceso [3].

En la presente investigación, se establecerá una metodología de diseño de un taladro de árbol para madera. El artículo se estructura así: primero se establecen los materiales y métodos para el diseño; luego se modelan matemáticamente las fuerzas de trabajo de los actuadores neumáticos; y finalmente se realiza la propuesta de diseño.

\section{Materiales y métodos}

Inicialmente, se describe de manera detallada el proceso actual del mecanizado de agujeros y sus respectivas fases de operación. Es decir, para el desarrollo del diseño se tuvieron en cuenta las diferentes fases u operaciones que debe realizar el operario de manera manual.

En primera instancia el operario se debe desplazar hacia un punto en donde se encuentra la materia prima; seguidamente, y dependiendo el diámetro y la longitud, debe ubicar la posición donde se realizará el agujero; luego se traslada a la zona de mecanizado donde se sujeta, y a través de una palanca procede a mecanizar el agujero. Finalmente, el operario debe marcar la madera con el fin de que la persona que vaya a instalar el parque, logre identificar la pieza a la hora de ensamblar, figura 4 .

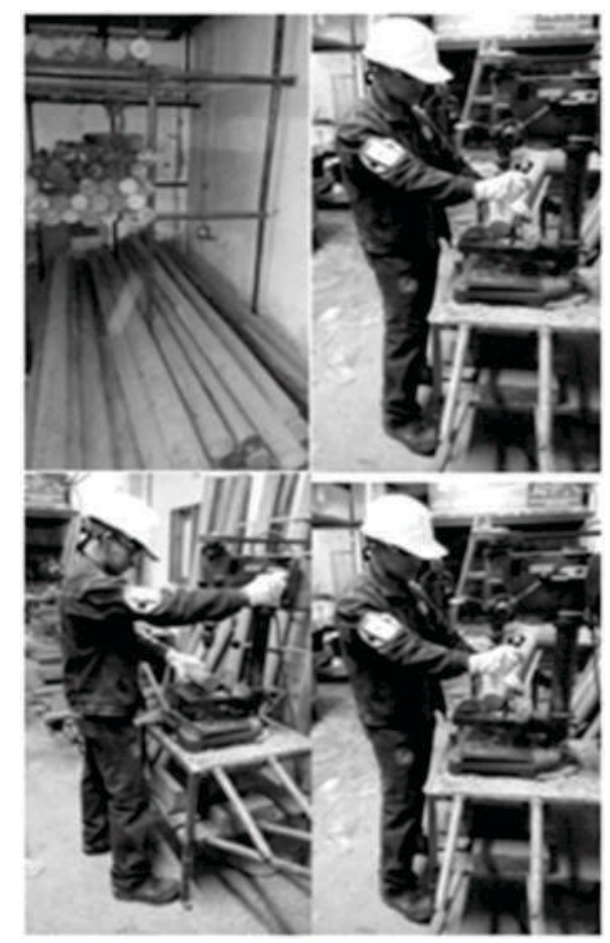

Figura 4. Mecanizado actual del proceso de taladrado.

Fuente: elaboración propia, empresa. Parques Infantiles EBATEC S.A.S

Teniendo en cuenta las fases anteriormente descritas, se procedió a caracterizar cada uno de los procesos inmiscuidos buscando las posibles soluciones hacia un sistema automatizado; para ello se tuvieron en cuenta los diferentes esfuerzos a los que están sometidos los elementos de cada fase, y así determinar el tipo de energía con la que se pretende automatizar la máquina.

Actualmente existen cuatro tipos de energías para automatizar un proceso de esta naturaleza: la 
neumática, la hidráulica, la mecánica y la eléctrica. Cada una de ellas tiene su campo de acción dependiendo de la magnitud de la fuerza que se requiera utilizar durante el proceso. Al convertirlo en trabajo, este lo deberán realizar los diferentes actuadores, entendiéndose que un actuador es aquel dispositivo que convierte algunos de los tipos de energía nombrados anteriormente en trabajo. Teniendo en cuenta las fuerzas a generar, se opta por comparar la energía neumática e hidráulica, [4].

\begin{tabular}{|c|c|c|}
\hline & Neumática & Hidráulica \\
\hline $\begin{array}{l}\text { Estado de las } \\
\text { fugas }\end{array}$ & $\begin{array}{l}\text { Solo perdida } \\
\text { de energía }\end{array}$ & Contaminación \\
\hline $\begin{array}{l}\text { Influencia } \\
\text { Ambiental }\end{array}$ & $\begin{array}{l}\text { A prueba de } \\
\text { explosión. } \\
\text { Insensible a la } \\
\text { temperatura }\end{array}$ & $\begin{array}{l}\text { Riesgo de } \\
\text { incendio en caso } \\
\text { de fuga. Sensible } \\
\text { a cambios de } \\
\text { temperatura. }\end{array}$ \\
\hline $\begin{array}{l}\text { Almacenaje } \\
\text { de energía }\end{array}$ & Fácil & Limitada \\
\hline $\begin{array}{l}\text { Transmisión } \\
\text { de energía }\end{array}$ & $\begin{array}{l}\text { Velocidad de la } \\
\text { señal } 20 \quad-40 \\
\mathrm{~m} / \mathrm{s}\end{array}$ & $\begin{array}{l}\text { Velocidad de la } \\
\text { señal hasta } 1000 \\
\mathrm{~m} / \mathrm{s}\end{array}$ \\
\hline $\begin{array}{l}\text { Velocidad de } \\
\text { Operación }\end{array}$ & $V=1.5 \mathrm{~m} / \mathrm{s}$ & $V=0.5 \mathrm{~m} / \mathrm{s}$ \\
\hline $\begin{array}{l}\text { Coste de } \\
\text { Alimentación }\end{array}$ & Muy Alto & Alto \\
\hline $\begin{array}{l}\text { Movimiento } \\
\text { Lineal }\end{array}$ & $\begin{array}{l}\text { Simple con } \\
\text { cilindros. } \\
\text { Fuerzas } \\
\text { limitadas. } \\
\text { Velocidad } \\
\text { dependiente de } \\
\text { la carga. }\end{array}$ & $\begin{array}{l}\text { Simple con } \\
\text { cilindros. Buen } \\
\text { control de } \\
\text { Velocidad. } \\
\text { Fuerzas muy } \\
\text { grandes. }\end{array}$ \\
\hline $\begin{array}{l}\text { Movimiento } \\
\text { giratorio }\end{array}$ & $\begin{array}{l}\text { Simple. } \\
\text { ineficiente, alta } \\
\text { velocidad }\end{array}$ & $\begin{array}{l}\text { Simple. par alto, } \\
\text { baja velocidad }\end{array}$ \\
\hline $\begin{array}{l}\text { Exactitud de } \\
\text { posición }\end{array}$ & $\begin{array}{l}1 / 10 \mathrm{~mm} \\
\text { posible sin } \\
\text { carga }\end{array}$ & $\begin{array}{l}\text { Puede } \\
\text { conseguirse } 1 \\
\text { mm }\end{array}$ \\
\hline Estabilidad & $\begin{array}{l}\text { Baja, el aire es } \\
\text { compresible }\end{array}$ & $\begin{array}{l}\text { Alta, ya que el } \\
\text { aceite es casi } \\
\text { incompresible, } \\
\text { además el nivel } \\
\text { de presión es } \\
\text { más alto que en } \\
\text { el neumático }\end{array}$ \\
\hline Fuerzas & $\begin{array}{l}\text { Protegido } \\
\text { contra } \\
\text { sobrecargas. } \\
\text { Fuerzas } \\
\text { limitadas por la } \\
\text { presión } \\
\text { neumática y el } \\
\text { diámetro del } \\
\text { cilindro } \\
\text { F=30KN a } 6 \\
\text { bar) }\end{array}$ & $\begin{array}{l}\text { Protegido contra } \\
\text { sobrecargas. con } \\
\text { presiones que } \\
\text { alcanzan los } 600 \\
\text { bar y pueden } \\
\text { generarse } \\
\text { grandes fuerzas } \\
\text { hasta } 3000 \mathrm{~N} \text {. }\end{array}$ \\
\hline
\end{tabular}

Tabla 1. Comparativo entre la Neumática y la Hidráulica [5].

Observando las características que brindan cada una de las energías, se opta por escoger la neumática ya que el tiempo de respuesta y precisión son dos características que posee a la hora de automatizar. Además, las fuerzas que se generan dentro del proceso están dentro del rango de trabajo de los actuadores los cuales comercialmente vienen de diferentes diámetros y carreras, lo que permite tener cierta flexibilidad a la hora de calcular y seleccionar los más apropiados para los diferentes procesos.

De otra parte, para el sistema de control se decide utilizar un PLC (Controlador Lógico Programable), ya que es un dispositivo electrónico digital que utiliza una memoria para almacenar instrucciones e implementar funciones específicas de lógica, secuencia, temporizado y sirve para controlar máquinas y procesos, [6]

\subsection{Actuadores neumáticos.}

Los actuadores son aquellos que realizan directamente el trabajo dentro de un sistema automático o semiautomático. Los tipos de actuadores están construidos según las características propias de la aplicación, [7].

Las características genéricas de un actuador son:

a) Principio operativo (doble efecto - simple efecto) b) Diámetro del émbolo c) Carrera de desplazamiento Un actuador neumático del tipo cilíndrico está compuesto de los siguientes elementos, figura 5 :

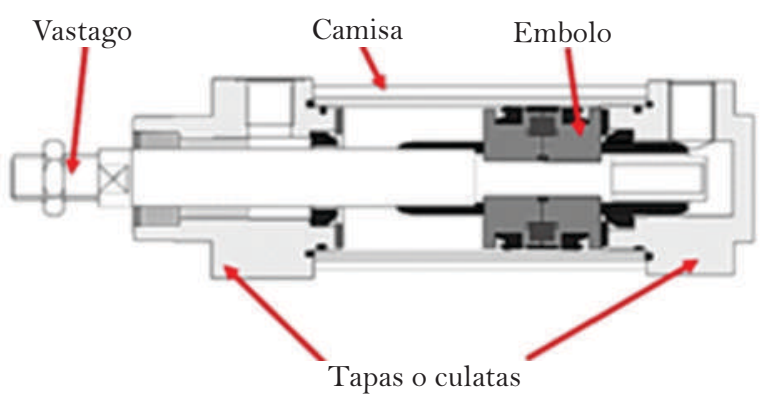

Figura 5. Actuador neumático [7].

\subsubsection{Modelamiento de la fuerza de trabajo de los actuadores neumáticos.}

El diámetro del émbolo determina la fuerza que puede desarrollar el actuador. Como la presión es la fuerza sobre unidad de área (1):

$$
P=\frac{F}{A}
$$

Donde:

\section{$P=$ Es la presión en bar}

\section{$F=$ Es la fuerza en newton}

$A=$ Es el área en $\mathrm{cm}^{2}$

Y la fuerza es dependiente directamente de la presión y del área; y debido a que normalmente las máquinas manejan una presión constante (6 bar, estimado), es entonces que la fuerza que puede realizar un actuador neumático depende de la geometría y diámetro de su émbolo. 

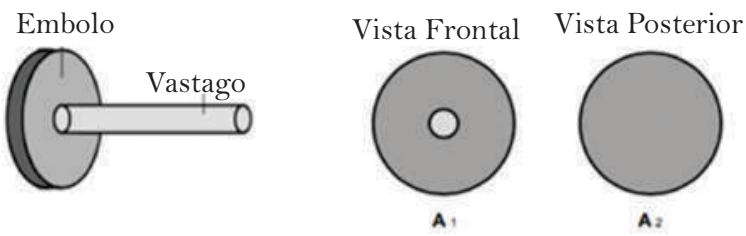

Figura 6. Embolo y Vástago de un actuador neumático [7].

Las áreas del émbolo de avance y de retroceso son diferentes.

$$
\text { A1 }<\text { A2 }
$$

Por lo tanto, se concluye simplemente que la fuerza de avance es mayor que la fuerza de retroceso en un actuador de doble efecto, con una misma presión de trabajo.

\section{$\mathrm{F}$ avance $>\mathrm{F}$ retroceso}

La diferencia de áreas es debida al área que ocupa el vástago en el émbolo.

\subsection{Controlador Lógico Programable (PLC).}

Es un dispositivo que tiene la función de ser el cerebro de un proceso automatizado. Es decir, es el encargado de controlar paso por paso las funciones de cada uno de los implementos mencionados anteriormente con la finalidad de realizar los objetivos propuestos en su programación etapa por etapa para así darle autonomía a determinada máquina. Es un elemento usado industrialmente ya que está adaptado para soportar cualquier tipo de ambiente laboral, como altas temperaturas, vibraciones, ruido eléctrico, entre otros. Es por esto que siempre se tiene en cuenta en procesos de producción en industrias, como también atracciones de parques y más. [5]

Una de las características que tiene el PLC, figura 7, es que no solo recibe y envía ordenes, sino que también estudia y verifica las señales que emite la maquina a controlar con las instrucciones de su programa, verificando que todo este marchando bien sin riesgo de que haya una desestabilización.

Básicamente el proceso que realiza es el de leer las entradas y salidas y conforme a los datos que estas contengan realiza la ejecución de su programa, primeramente, examina los datos que llegan a sus entradas, seguido de esto escoge la decisión correcta de acuerdo a los parámetros de su programa, almacenando los datos recibidos y realizando sus respectivos cálculos. Finalmente dependiendo de los resultados que obtenga en su análisis, envía las órdenes a los elementos actuadores, [5].

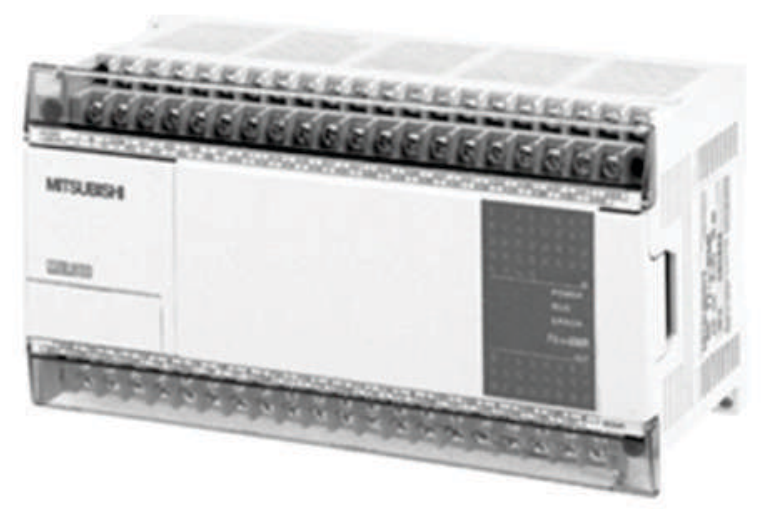

Figura 7. PLC de corriente continua de Mitsubishi (FX1N-60MR-D) [8].

\section{Diseño del Sistema Propuesto}

Para la realización del diseño se tuvieron en cuenta las diferentes estaciones con el fin de poder integrarlas, y que el operario sólo se limitara a alimentar la máquina con los maderos a trabajar, y a su vez supervisa los movimientos del proceso de manufactura. A continuación, se describirán dos propuestas y se hará un breve análisis para cada uno de ellos.

El primer diseño propuesto se observa en la Figura 8, la cual integra un sistema de corte que utiliza dos mordazas para sujetar el madero, una vez un sensor detecte el madero permitiendo que el disco de corte realice su trabajo., las mordazas se accionarán a través de actuadores que tiene ubicado un sensor de presión que permite sujetar el madero con una determinada fuerza; cuando el sensor de presión llegue a su set point enviará una señal al PLC informando que el madero ya está listo para ser cortado. Realizado el corte las prensas se abren permitiendo que el madero se desplace hasta la estación de perforado, la cual lleva el mismo sistema de la estación de corte, una vez el sensor indique la posición del madero se procede a perforar los dos agujeros, finalmente el madero llega a la estación de marcado donde dos matrices que a través de actuadores son accionados para realizar el nombrado de los maderos con logo y número de madero. 


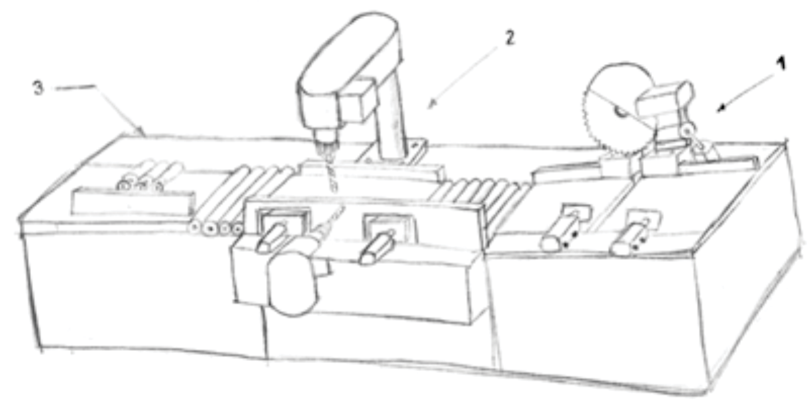

Figura 8: Primer boceto realizado para el diseño del taladro de árbol automatizado. Fuente: elaboración propia.

Para el segundo boceto propuesto, mostrado en la Figura 9, se observa que el sistema de alimentación es diferente con respecto al primero. En la primera propuesta se muestra un sistema de rodillos que lleva el madero a cada una de las estaciones, para la propuesta dos se decide utilizar un sistema de poleas, que llevara la madera en el aire con el fin de evitar la fricción que se presenta en la anterior propuesta, una de las poleas es movible en sentido horizontal con el fin de que el sistema se adapte según sea el diámetro de la madera, la otra polea es fija y esta sujetada a un motor paso a paso, lo que permitirá manejar con precisión la posición de cada una de los maderos antes de ser cortado, perforado y marcado. Para cada uno de los procesos se contará con los sensores más adecuados que permitirán enviar la información al PLC.

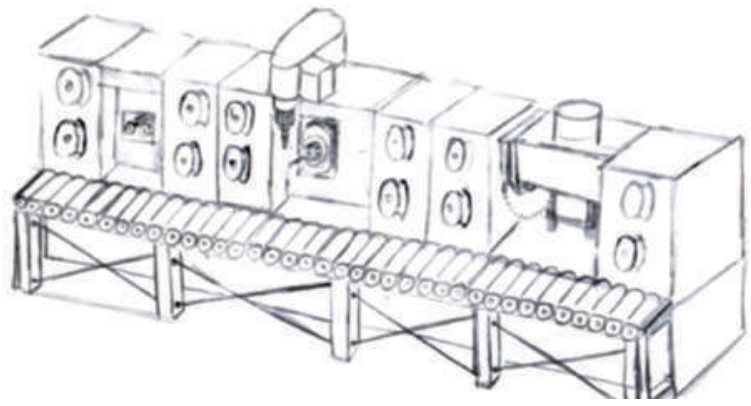

Figura 9: Segundo boceto realizado para el diseño del taladro de árbol automatizado, Fuente: elaboración propia.

Una vez descritos ambos procesos, se determina que el segundo boceto es el de mejor comportamiento debido a que su sistema de alimentación es mucho más seguro y preciso. Además, se podrá trabajar diferentes diámetros y longitudes de maderos, lo cual implica que el controlador lógico programable deberá almacenar una secuencia de trabajo para cada uno de los maderos. La máquina tendrá la capacidad de almacenar el trabajo individual de cada uno de los maderos en cada uno de los parques infantiles requeridos.

A través de una pantalla HMI el operario será el encargado de seleccionar el programa del madero a trabajar, teniendo en cuenta que para cada diámetro y cada longitud se deberán tener sensores que ayuden al posicionamiento con el fin de mecanizar los agujeros.

Los avances de las brocas son realizados por un actuador oleo-neumático, el cual garantiza la velocidad constante y la uniformidad del proceso de mecanizado. A continuación se muestra el detalle del sistema de alimentación para la propuesta escogida. Figura 10
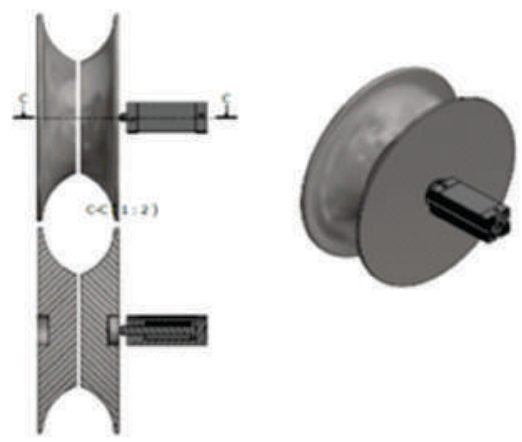

Figura 10: Diseño del sistema de alimentación Fuente: elaboración propia.

Teniendo que para el sistema de alimentación, la selección del diámetro del embolo del actuador viene dado por:

$$
d=\sqrt{\frac{M_{\text {total }} \cdot g}{\frac{\pi}{4} \cdot P_{\text {aire }}}}
$$

Donde:

$M_{\text {total }}$ : Masa total del conjunto (Polea, rodamiento, madera)

$g$ : Constante aceleración de la gravedad

$P_{\text {aire }}:$ : Presión del aire

\section{Conclusiones}

En el desarrollo del proyecto de diseño se identificó que, mediante la caracterización de las diferentes estaciones o procesos del mecanizado de agujeros, se logra determinar el funcionamiento de cada una de las partes que componen el sistema, adicionalmente se logró identificar las falencias con mayor criticidad a los cuales se pudo encontrar la solución teórica y práctica. 
Mediante la investigación de diferentes dispositivos, se puede adaptar dentro de la máquina mecanismos que permitirán el correcto funcionamiento, hasta tal punto de corregir todas las inconsistencias y mejorar significativamente la precisión de la máquina y el tiempo de ejecución del proceso, adicionalmente los dispositivos adquiridos tienen la versatilidad de cambiar, posición y fuerza a través de sensores de posición y de presión.

Al diseñar el sistema de alimentación, sujeción, corte, taladrado y marcado de las piezas se puede observar que con esto se pudo integrar todo en una misma máquina, obteniendo tiempos cortos de mecanizado, confort para el operario y un aumento en la productividad.

\section{Reconocimientos}

A la empresa EBATEC S.A.S, por acceder a realizar las pruebas y mediciones de diseño mostradas.

\section{Referencias}

[1] A. J. Molina, "Diseño de una máquina automatizada perforadora de tablas de madera”, tesis, Universidad Simón Bolívar, Sertenejal, Venezuela, 2006.

[2] Y. Ríos, "Automatización de un taladro para perforar papel”, tesis, Instituto Politécnico Nacional, México, 2008.

[3] J. A. López, "Diseño de un sistema de control para un taladro electro- neumático de banco, bajo norma gemma y ambiente scada, con seguimiento a ciclos de operación”, tesis, Universidad Tecnológica de Pereira, Colombia, 2014.

[4] G. Maldonado," Mecatrónica, automatización Studio", 2011. [En línea]. D i s p o n i b l e e n : https://es.slideshare.net/GaloMaldonado /mecatrnica-automatizacin-y-automationstudio-10159025.

[5] Automation Studio, "Mecatrónica, Automatización y automation studio", 2011. [En línea]. Disponible en: https://es.slideshare.net/GaloMaldonado / mecatrnica-automatizacion-yautomation-studio-10159025.
[6] P. Prieto, "MONOGRAFICO: lenguajes de programación-Principios básicos de PLC", 2007. [En línea]. Disponible en: http://recursostic.educacion.es/observatorio/web /gl/component/content/article/502monografico-lenguajes-de-programacion? start $=2$

[7] P. Croser. F. Ebel, "Pneumatics. Basic Level”. Denkendorf: Festo Didactic, 2002, pp. 34-38.

[8] Mitsubishi Electric, "Programmable controllers", 2015. [En linea]. Disponible e $\mathrm{n}$ :

http://dl.mitsubishielectric.com/dl/fa/do cument/manual/plc_fx/jy992d89301/jy9 92d89301r.pdf 